\title{
Social vulnerability, parity and food insecurity in urban South African young women: the healthy life trajectories initiative (HeLTI) study
}

\author{
Lisa J. Ware ${ }^{1,2}$ (D) Andrew W. Kim ${ }^{1,3}$. Alessandra Prioreschi ${ }^{1}$. \\ Lukhanyo H. Nyati ${ }^{1}$. Wihan Taljaard ${ }^{1}$. Catherine E. Draper ${ }^{1}$. Stephen J. Lye ${ }^{4,5}$. \\ Shane A. Norris ${ }^{1,6}$
}

Accepted: 29 April 2021 / Published online: 19 May 2021

○ The Author(s), under exclusive licence to Springer Nature Limited 2021

\begin{abstract}
Social vulnerability indices (SVI) can predict communities' vulnerability and resilience to public health threats such as drought, food insecurity or infectious diseases. Parity has yet to be investigated as an indicator of social vulnerability in young women. We adapted an SVI score, previously used by the US Centre for Disease Control (CDC), and calculated SVI for young urban South African women $(n=1584$; median age 21.6 , IQR 3.6 years). Social vulnerability was more frequently observed in women with children and increased as parity increased. Furthermore, young women classified as socially vulnerable were 2.84 times (95\% CI 2.10 $3.70 ; p<0.001)$ more likely to report household food insecurity. We collected this information in 2018-2019, prior to the current global COVID-19 pandemic. With South Africa having declared a National State of Disaster in March 2020, early indicators suggest that this group of women have indeed been disproportionally affected, supporting the utility of such measures to inform disaster relief efforts.
\end{abstract}

Keywords Vulnerable populations · Parity · Urban health $\cdot$ Young adult · Disaster planning $\cdot$ Social Protection $\cdot$ Women

\section{Introduction}

'Social vulnerability' describes the vulnerability of communities and individuals to destructive hazards, most frequently natural disasters, and is typically assessed using a series of social, economic and environmental indicators such as employment, education, population density, geographical location and age [1-3]. Social vulnerability indices for Africa have further considered the proportion of people living with HIV

Lisa J. Ware

lisa.ware@wits.ac.za

Extended author information available on the last page of the article 
and the rate of urbanisation alongside national indicators for health expenditure, trade and corruption [4]. In this context, high levels of social vulnerability increase susceptibility to drought [4], food insecurity [5] and reduce resilience to infectious disease $[6,7]$.

Women of reproductive age in South Africa face additional social and physical vulnerabilities that could increase susceptibility or reduce resilience to public health threats. They are vulnerable to traumatic events, intimate-partner and gender-based violence, and at higher risk for HIV infection, depression and pregnancy [8-11]; half to two thirds of their pregnancies are not planned [12, 13]. Previous studies suggest that both unplanned pregnancy and higher parity increase vulnerability to household food insecurity $[14,15]$.

The aims of this study were (1) to determine if parity is a predictor of social vulnerability in a large random sample of young women from an urban African township and (2) assess the relationships between parity, health, social vulnerability and household food insecurity.

\section{Methods}

This cross-sectional study of young women formed part of the World Health Organization (WHO) Healthy Life Trajectories Initiative (HeLTI), with intervention cohorts in Canada, China, India and South Africa [16-18]. The primary aim of HeLTI was to examine the impact of a complex continuum of care intervention beginning in the preconception period on maternal and child health to offset obesity risk in early childhood. We recruited women (June 2018 to July 2019) from randomly selected areas of Soweto using $k$-means clustering to define thirty communities with a $1 \mathrm{~km}^{2}$ radius each in such a way as to minimise the sum of squares within each cluster [19]. The Human Research Ethics Committee (Medical) at the University of the Witwatersrand approved the study (M171137, M1811111).

Fieldwork teams visited households to record type of residence (formal or informal), household density and the number of household assets. Eligible women from the household (ages 18.0-25.9 years; not pregnant, and no previous diagnosis of cancer, Type I Diabetes, or Epilepsy) attended the research unit in Soweto for an intervieweradministered survey and physical measurements. Survey domains included (1) sociodemographic (education, employment, relationships); (2) general health (medical and reproductive history, disease and medication use, HIV status, tobacco and alcohol use); (3) mental health (stressful life events, depression and anxiety); and (4) food insecurity. Survey questions used the WHO STEPS protocol, the United States (US) Centre for Disease Control (CDC) Global Adult Tobacco Survey [20], the WHO Alcohol Use Disorders (WHO-AUDIT) Test [21], the Adverse Childhood Experiences (ACE) Questionnaire [22], the PHQ-9 (score of 0-27; cut off $\geq 10$ applied for probable depression) [23], and the General Anxiety Scale (GAD-7; score of 0-21; cut off $\geq 10$ indicating moderate to severe symptoms) [23]. We recorded cell phone and email access using the questions: " Do you currently have: (a) Your own number you can always be contacted on (cell phone); and (b) Your own email address that you are able to check regularly?". We assessed food insecurity using an adapted Community Childhood Hunger 
Identification Project (CCHIP) index [24]. Physical measurements included blood pressure and anthropometry (height, weight and waist circumference (central adiposity)); all measured in triplicate and following WHO training on standardisation [25, 26] and International guidelines [27]. We collected and managed study data using REDCap electronic data capture tools [28]. Deidentified data sharing is available upon request, please contact Lisa Ware.

\section{Social vulnerability index (SVI)}

We calculated SVI score for each participant using a similar set of composite measures to those previously used by the CDC [29] across four domains: (1) socioeconomic status (SES: income, poverty, employment and education); (2) household composition and disability (age, single parenting and disability); (3) housing and transportation (housing structure, crowding and vehicle access) and (4) minority status and language (race, ethnicity and English-language proficiency).

\section{Socioeconomic status}

We replaced income and poverty with household asset score from a list of 13 common assets (electricity, fridge, stove, vacuum cleaner, washing machine, satellite TV, DVD player, car, TV, landline telephone, cell phone, computer/laptop/tablet and internet access), shown to be central in economic assessment of the household and sensitive to change over time [30, 31]. We recorded unemployment for participants not currently working or studying and assessed education as the total number of years in education.

\section{Household composition and disability}

We reviewed ages of household residents for vulnerable age groups $(<18$ or $\geq 65$ years). We did not use single parenting ( $\geq 1$ child and single or not living with partner) so as not to bias results in this analysis. We recorded disability if participants reported claiming employment disability support.

\section{Housing and transportation}

We recorded housing structures as formal (houses or apartments made of construction materials such as brick or concrete) or informal (shacks or containers). We determined crowding or household density by the number of household residents that stayed in the home most nights over the last 3 months, divided by the number of rooms available for sleeping. We assessed transport access by whether the household had a motorcar.

\section{Minority status and language}

We did not employ these indicators as Soweto is predominantly black African ethnicity $(98.5 \%)$ with the majority of inhabitants speaking at least one of the five 
mainly used African languages in the area and very few (2.3\%) speaking English as a first language [32].

We calculated the SVI score as a count of the number of individual variables meeting the criteria in Table 1 to generate a score between zero and eight. This process identified individuals as socially vulnerable if their SVI score was in the highest 90 th percentile.

\section{Statistical analysis}

We compared median continuous variables using independent-samples Kruskal-Wallis test with Dunn-Bonferroni post-hoc pairwise comparisons and categorical data compared using Pearson Chi square test. We used Spearman partial correlation to test if there was an association between continuous or ordinal variables, or both, while controlling for age. We applied logistic regression to determine the effect of parity, age, and maternal age at first birth on SVI classification, and multinomial logistic regression to assess whether SVI predicted household food insecurity. For all analysis, we used IBM SPSS Statistics for Windows, Version 25.0 (IBM Corp, Armonk, NY).

\section{Results}

Of the 1719 young women attending the research unit (Fig. 1), 1584 had complete data for parity, health, and SVI indicators. Of these, $808(51 \%)$ were nulliparous (had not yet given birth to a live child), 645 (41\%) were primiparous and $131(8 \%)$ were multiparous. Table 2 compares sociodemographic data,

Table 1 Social vulnerability domains and indicators used

\begin{tabular}{|c|c|}
\hline Domain and indicator & Social vulnerability recorded if \\
\hline \multicolumn{2}{|l|}{ A.Socioeconomic status } \\
\hline 1. Sum of total household assets $(0-13)$ & In the lowest 10 th percentile ( $\leq 5 \mathrm{HH}$ assets) \\
\hline 2. Employment & Currently unemployed (excluding students) \\
\hline 3. Education (number of total years) & In the lowest 10 th percentile ( $\leq 11$ years) \\
\hline \multicolumn{2}{|l|}{ B. Household composition and disability } \\
\hline 4. Household age & $\mathrm{HH}$ residents $<18$ or $\geq 65$ years in age \\
\hline 5. Disability & Claiming employment disability allowance \\
\hline \multicolumn{2}{|l|}{ C. Housing and transportation } \\
\hline 6. Housing type & Informal (shack or container) \\
\hline $\begin{array}{l}\text { 7. Household density (household residents/ } \\
\text { number of rooms for sleeping) }\end{array}$ & In the highest 90 th percentile ( $\geq 5$ per room $)$ \\
\hline 8. Transport & Household does not own a car \\
\hline SVI Score & $\begin{array}{l}\text { One point is allocated for each of the criteria met above } \\
\text { creating scores ranging from } 0-8\end{array}$ \\
\hline "Socially vulnerable" if: & SVI score in the highest 90th centile for the group $(\geq 4)$ \\
\hline
\end{tabular}


health and resources by parity. Young women with children were significantly older than those without children, with age increasing as parity increased and multiparous young women were more likely to have had their first child at a younger age. Nulliparous women more frequently held a high school certificate and were more likely to be enrolled in education at the time of data collection. When adjusted for age, a negative linear relationship between number of children and years of education remained $\left(r_{s}(1580)=-0.245, p<0.001\right)$. Access to social grants was significantly higher in women with children as may be expected through use of child support grants. Cell phone access was high across all groups $(>90 \%)$ but access to a personal email address was significantly lower

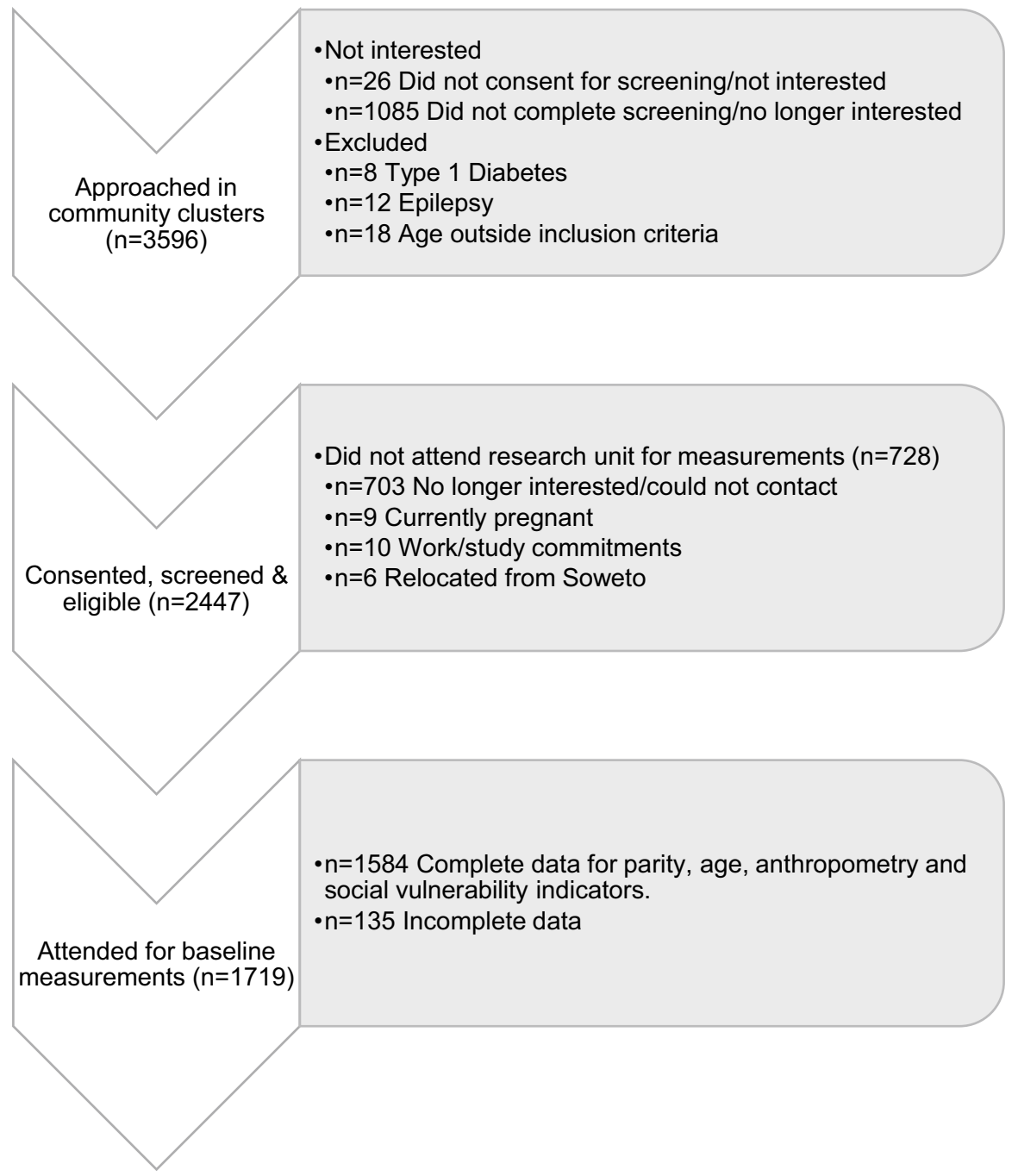

Fig. 1 Study flow diagram - recruitment and inclusion of young women age 18.0-25.9 years 


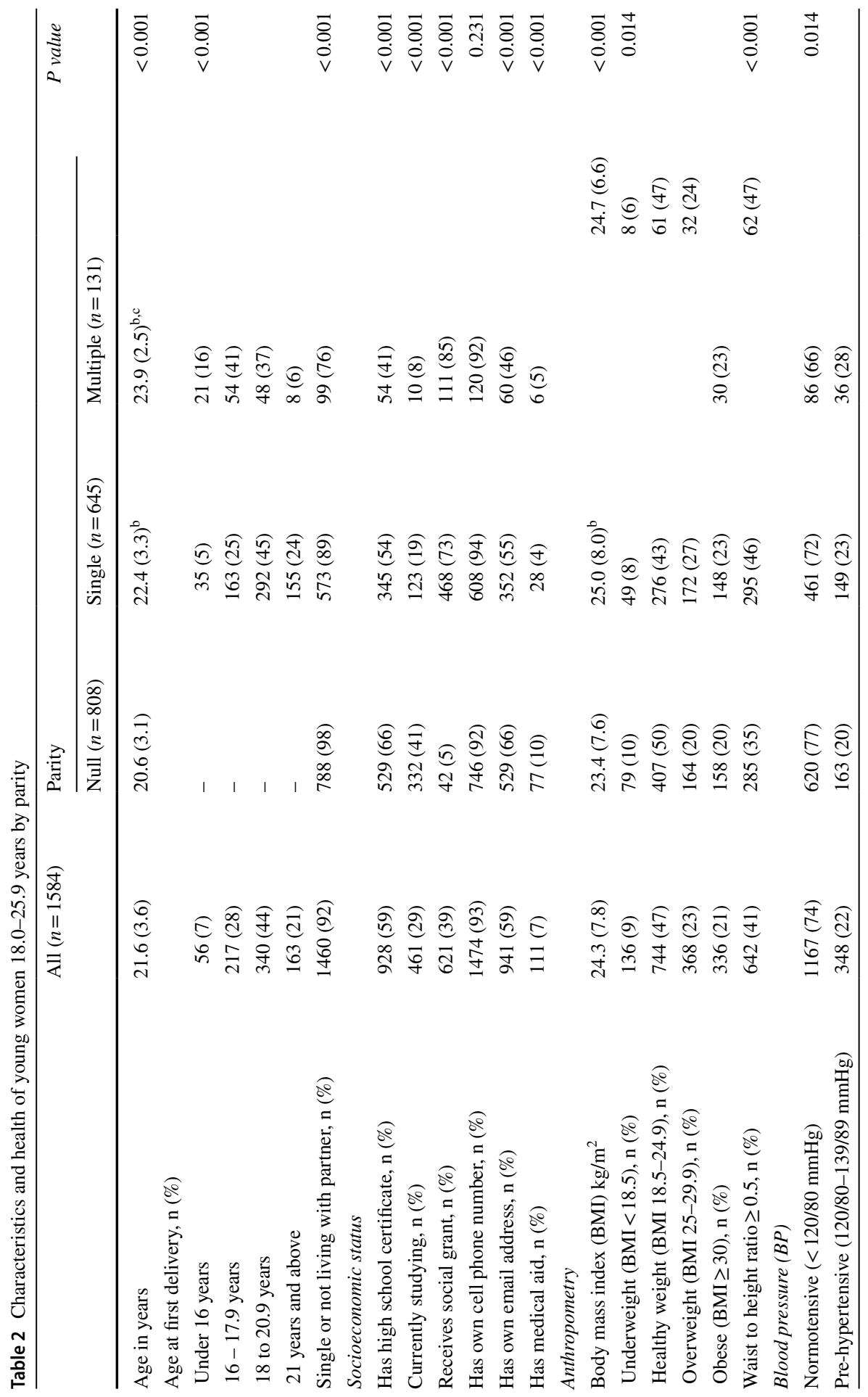

尔 


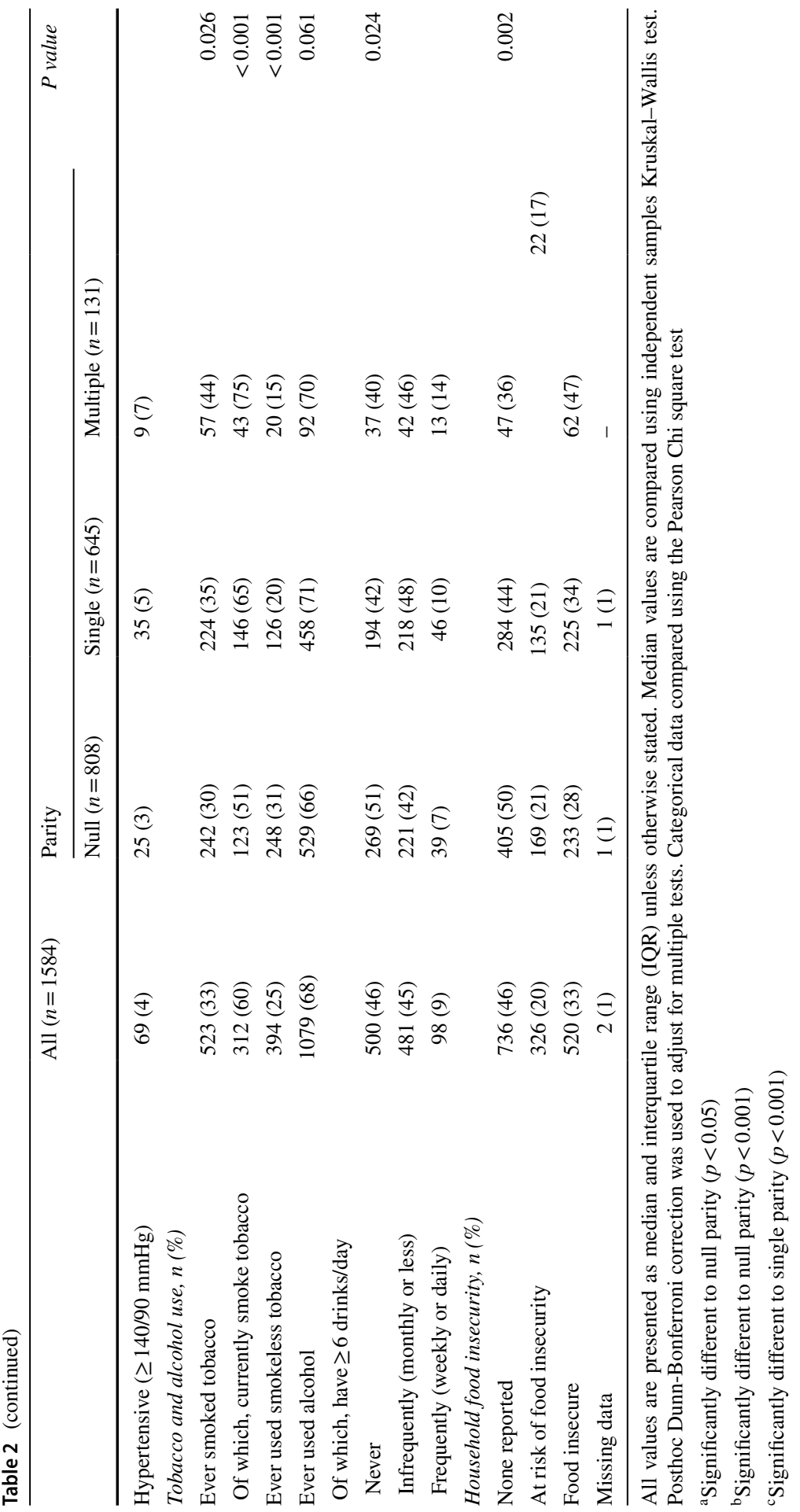


in women with children. Medical aid coverage was low across all groups $(<10 \%)$ and significantly lower in women with children $(\leq 5 \%, p<0.001)$. Comparing the health of the three groups, women with children were more frequently overweight or obese. When adjusting for age, the relationship between the number of children and all markers of weight status, including BMI $\left(r_{s}(1581)=0.054\right.$, $p=0.032)$, waist circumference $\left(r_{s}(1581)=0.097, p<0.001\right)$ and waist-toheight ratio $\left(r_{s}(1581)=0.098, p<0.001\right)$ remained significant, although the association was very weak. Young women with children were also significantly more likely to be classified as prehypertensive or hypertensive based on blood pressure readings or use of antihypertensive medication, or both. Women with children were significantly more likely to have been tested for HIV (as this is part of routine antenatal care) though self-reported rates of testing positive did not differ significantly by group, with $5 \%$ of young women reporting a positive status (data not shown). Current cigarette smoking was significantly higher in women with children, while smokeless tobacco use was significantly higher in nulliparous young women and alcohol use prevalent across all groups.

Reported ACEs and all measures of psychological health did not differ by parity. Overall, 19\% of women reported moderate to severe scores for depression (PHQ-9 score $>10$ ); $15 \%$ reported moderate to severe scores for anxiety (GAD-7 score $>10) ; 55 \%$ of women reported four or more ACEs; $39 \%$ reported $1-3$ and only $6 \%$ reported no ACEs (data not shown). Food insecurity was present in one third of households, with a further $21 \%$ of households at risk of food insecurity. Women with children were significantly more likely to experience food insecurity in the household, with food insecurity present in almost half (47\%) of the multiparous young women's households.

With the exception of disability (low across all groups), young women with children were significantly more likely to meet the criteria for each of the SVI indicators (Table 3). Almost half of the members of the multiparous group (49\%) classified as socially vulnerable, compared to $31 \%$ of the primi- and $16 \%$ of the nulliparous group $(p<0.001)$. Logistic regression models showed that it was parity and not age or maternal age at first birth that was associated with social vulnerability (Table 4); multiparous young women had 5.05 higher odds of being classified as socially vulnerable compared to their peers without children. Additional potential vulnerability indicators showed more women with children shared toilet facilities with other households (multi- 63\%; primi- 56\%; nulliparous $48 \%$; $p=0.001$ ), and fewer had a piped drinking water supply inside the home (multiparous 35\%; primiparous 44\%; nulliparous 49\%; $p=0.001$ ).

Young women classified as socially vulnerable were 2.84 times (95\% CI $2.10-3.70 ; p<0.001)$ more likely to report food insecurity and 1.55 times $(95 \%$ CI 1.13-2.14; $p=0.007$ ) more likely to report being at risk of food insecurity in the household. Furthermore, compared to young women not classified as socially vulnerable, those who were classified as socially vulnerable using this score were 2.7 times (95\% CI 2.12-3.46; $p<0.001$ ) more likely to have ever been pregnant. 


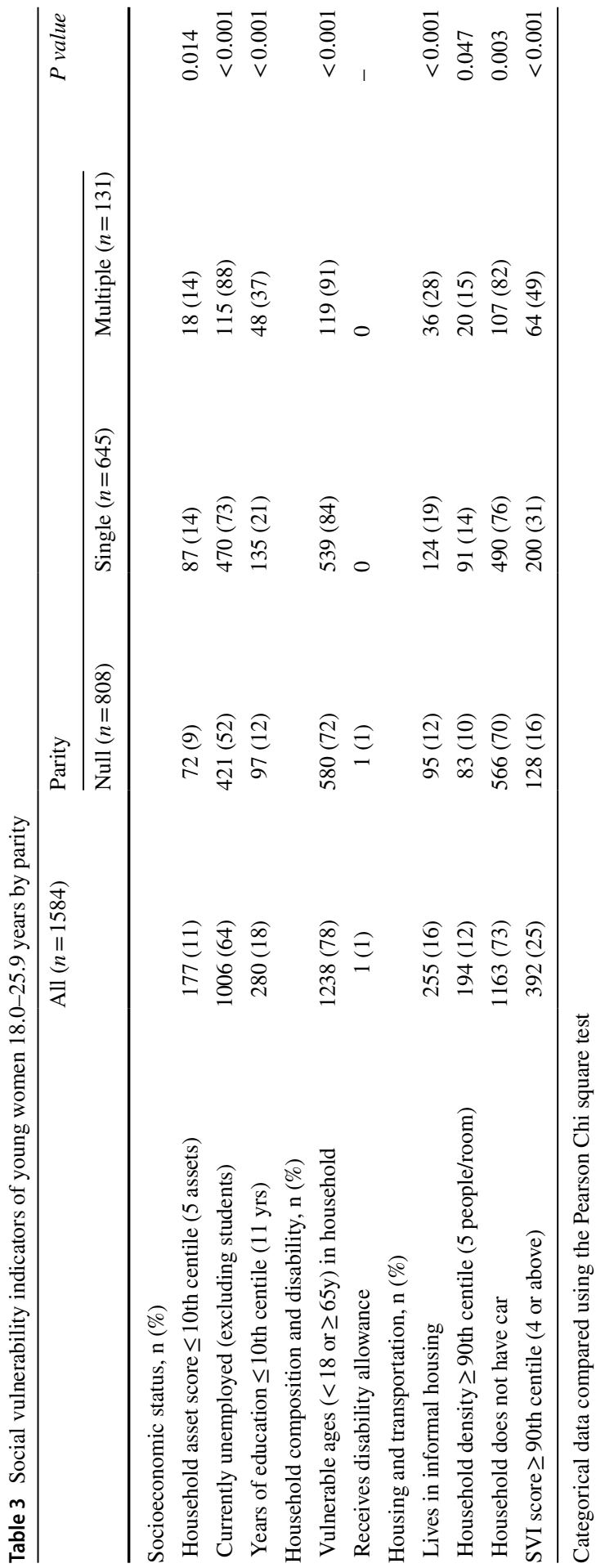


Table 4 Binomial logistic regression predicting likelihood of social vulnerability based on parity and age or maternal age at first birth

\begin{tabular}{lccccccc}
\hline \multicolumn{1}{c}{$\beta$} & SE & Wald & df & $p$ & Odds ratio & $95 \%$ CI \\
\hline All young women & $(n=1584)$ & & & & & & \\
Age & 0.002 & 0.031 & 0.003 & 1 & 0.954 & 1.00 & $0.94-1.06$ \\
Primiparous & 0.868 & 0.136 & 40.59 & 1 & $<0.001$ & 2.38 & $1.82-3.11$ \\
Multiparous & 1.619 & 0.218 & 55.42 & 1 & $<0.001$ & 5.05 & $3.30-7.73$ \\
Constant & -1.707 & 0.646 & 6.98 & 1 & 0.008 & 0.18 & \\
Primi- and multiparous young women only $(n=776)$ & & & & $0.88-1.01$ \\
Maternal age & -0.055 & 0.035 & 2.44 & 1 & 0.119 & 0.95 & $1.32-2.90$ \\
Multiparous & 0.669 & 0.202 & 11.02 & 1 & 0.001 & 1.95 & \\
Constant & 0.266 & 0.687 & 0.15 & 1 & 0.698 & 1.31 & \\
\hline
\end{tabular}

\section{Discussion}

This study aimed to characterise social vulnerability using a contextually adapted SVI score across a large random sample of young women in an urban African township and to determine associations with health, parity, and food insecurity. Overall, one quarter of all young women were classified as socially vulnerable, with these young women more frequently exposed to food insecurity within the household. Furthermore, young women with higher parity typically had higher vulnerability.

Social vulnerability has been described as a dynamic state resulting from a set of decisions made over long periods of time leading to social, economic, political, environmental, organisational, or technical influences that impact individuals', households', and communities' ability to respond to economic and health shocks [33]. Importantly, disaster response efforts that aim to restore the community to the state prior to disaster, only perpetuate vulnerability to the next disaster [33]. Our study found that over half of the young women were food insecure or at risk of food insecurity. In 2008, a survey in urban South Africa reported that $80 \%$ of households were food insecure [34], suggesting that food insecurity levels have remained high despite a number of policies enacted in the intervening years [35]. Examining indicators of social vulnerability to public health threats could identify areas for development of collaborative policy while directing efforts at those most vulnerable. Our finding that parity in young women may be one such indicator of vulnerability to food insecurity is supported by evidence from other LMICs [36, 37] and directly responds to the Sendai Framework (2015-2030) call to better understand vulnerability to public health threats [38]. Research is needed to establish relevance across LMICs, as the various factors driving social vulnerability and resilience are not universal $[39,40]$.

Pregnancy at a young age in South African women has been associated with many causal factors [41]. Our findings that multiparous young women more frequently experienced lower levels of education, employment and poor-quality housing suggest potential for both social causes and consequences of childbearing at younger ages. The finding that socially vulnerable young women were almost three 
times more likely to have ever been pregnant accentuates need for greater social protection of young women in urban townships.

The association between higher parity and poorer health outcomes, including higher levels of overweight and obesity, aligns with findings from urban centres across sub-Saharan Africa [42]. Food insecurity and obesity serve as potent risk factors for both acute and chronic disease across the life course, including diabetes, hypertension, cardiovascular and renal disease, asthma, depression and anxiety, among others [43-46]. Young women and girls in urban African townships are particularly vulnerable to infection as a result of social, physical and economic drivers [47]. As young women with higher parity more frequently lived in households sharing drinking water sources and toilet facilities, this could further increase vulnerability to infectious disease. Women already tend to face greater difficultly during disasters and recovery compared to men due to employment disparities, lower wages and family care responsibilities [48, 49].

Indeed, the COVID-19 pandemic in South Africa has shown these associations to be the reality, with an estimated three million job losses, of which two million were women in formal and informal employment, disproportionately impacting young, black women with lower education levels and driving food insecurity in womenheaded households [50]. In response to both United Nation recommendations to target women and girls in social assistance packages and the initial findings of disproportionate impacts of the pandemic on women's participation in the labour market, early policy recommendations included increasing the value of child support grants (CSG) per child (not per caregiver) and reinstating school feeding schemes [51].

To mitigate the economic effects of the pandemic, the South African Government in March 2020 announced a relief package including a temporary increase in existing grants (including the child support known as the caregiver grant) and introduction of a new social relief of distress (SRD) grant, nicknamed the 'COVID grant' for individuals not receiving any other grants. The grants were initially planned for a six-month period, May until October 2020 [52] and, while the COVID grant has been extended into 2021 (accessed predominantly by men), the caregiver grant (accessed predominantly by women) was terminated in October 2020, with each grant at the time having around seven million beneficiaries [53]. Initial assessments of ending the caregiver grant suggest a negative impact particularly for black women with children on household and child hunger [54].

Analysis of the effectiveness of the caregiver grant indicates that the government strategy to initially boost the CSG per child for one month, then to top up the grant only per caregiver for the remainder of the six-month period, may have been less effective for reaching the poorest households and targeting food poverty than the called for strategy of a continued CSG increase per child, at the same cost [55]. As the economy reopens, there are calls to improve social protections such as unemployment insurance for workers in the informal sector, increase the CSG per child, initiate a universal Basic Income Guarantee, and improve and expand food programmes, early childhood development centres and community childcare facilities [56]. Alongside much needed improvements in sexual and reproductive health services for adolescent girls and young women [57], additional measures should include coordinated (public-private-NGO) multisectoral efforts to support young 
women to access employment or education opportunities, thereby improving household resilience to future threats. Over the coming months and possibly years, longitudinal cohorts such as HeLTI may provide insights into how socially vulnerable young women in such environments are affected by and recover from the COVID-19 pandemic.

Our results should be viewed within the limitations of this study, including recruitment of young women only from one geographical area. However, the finding that parity in young women predicts social vulnerability and food insecurity is supported by research from other countries. Soweto is similar to many low-resource urban areas with numerous environmental hazards (including water, food and energy insecurity; proximity to industrial activity) and public health threats both past and present [58]. Use of a simple social vulnerability assessment in such communities could promote targeted protection efforts, while informing development of cohesive policies to address the wider social, economic, political, environmental and structural causes of vulnerability.

Another limitation is the cross-sectional analysis in this study: it remains unclear if parity flows from initial states of vulnerability and disadvantage, or disadvantage and vulnerability stem from the constraints of multiparity in young women. Further analysis of longitudinal data is needed, though it may be that both scenarios are to some degree correct, compounding the impact of childbearing at a young age. This should reinforce calls for improved contraceptive and reproductive health services for young women in the preconception period. Additional initiatives that may benefit young women include the planned South African (SA) Medical Research Council (SAMRC) social impact bond, an initiative to attract investors to support programmes promoting adolescent girls and young women's sexual and reproductive health and targeting the social challenges they face [59]. Indeed, many of the interventions proposed by the SAMRC share significant overlap with those employed in the WHO-HeLTI trial in South Africa [60], including HIV testing with support to link to care; monitoring frequency and type of contraception use; supporting safe pregnancies (including early presentation for foetal ultrasound assessments) and psychosocial and behavioural interventions for improved physical and mental health. Both the HeLTI trial and the proposed SAMRC social impact bond will be rigorously evaluated to understand what works best to support vulnerable young women and girls while helping to reduce policy-implementation gaps.

\section{Conclusion}

In young women from an urban African township, parity was associated with numerous indicators of social vulnerability and with increased exposure to food insecurity. Contextually relevant social vulnerability indices may aid identification of vulnerable populations in public heath crises, and guide localised responses to public health threats. As COVID-19 has proven in South Africa that young women with children from low-income households are frequently the most affected by, and potentially, the least able to recover from national disasters. This work supports continued calls for increasing child support grants per child, while expanding community 
food provision, education and childcare facilities. Further support for multisectoral efforts to get and keep young women in education, training and employment is also warranted.

Acknowledgements The South African Medical Research Council and the Canadian Institutes of Health Research supported this study. The authors acknowledge the young women who participated in the study. LJW is supported by the South African DSI/NRF Centre of Excellence in Human Development. AWK is supported by the National Science Foundation Graduate Research Fellowship and the Fogarty International Center and National Institute of Mental Health, of the National Institutes of Health under Award Number D43 TW010543. The content is solely the responsibility of the authors and does not necessarily represent the official views of the National Institutes of Health, DSI or NRF.

\section{Declarations}

Conflict of interest The authors declare that there are no conflicts of interest.

\section{References}

1. Fekete A. Social vulnerability (Re-) assessment in context to natural hazards: review of the usefulness of the spatial indicator approach and investigations of validation demands. Int J Disaster Risk Sci. 2019;10(2):220-32.

2. Singh SR, Eghdami MR, Singh S. The concept of social vulnerability: a review from disasters perspectives. Int J Interdiscip Multidiscip Stud. 2014;1(6):71-82.

3. Aitsi-Selmi A, et al. The Sendai framework for disaster risk reduction: renewing the global commitment to people's resilience, health, and well-being. Int J Disaster Risk Sci. 2015;6(2):164-76.

4. Vincent K. Creating an index of social vulnerability to climate change for africa. Tyndall Cent for Clim Change Res Working Paper. 2004; 56(41). http://www.nrel.colostate.edu/ftp/conant/SLMknowledge_base/Vincent_2004.pdf.

5. Bohle HG, Downing TE, Watts MJ. Climate change and social vulnerability: toward a sociology and geography of food insecurity. Glob Environ Chang. 1994;4(1):37-48.

6. Kienberger S, Hagenlocher M. Spatial-explicit modeling of social vulnerability to malaria in East Africa. Int J Health Geogr. 2014;13(1):29.

7. Mathai MA. Sexual decision-making and AIDS in Africa: a look at the social vulnerability of women in sub-Saharian Africa to HIV/AIDS: a Kenyan example. Kassel: Kassel University Press $\mathrm{GmbH} ; 2006$.

8. Fonck K, et al. Increased risk of HIV in women experiencing physical partner violence in Nairobi, Kenya. AIDS Behav. 2005;9(3):335-9.

9. Dunkle KL, et al. Prevalence and patterns of gender-based violence and revictimization among women attending antenatal clinics in Soweto, South Africa. Am J Epidemiol. 2004;160(3):230-9.

10. Tsai AC, et al. Intimate partner violence and depression symptom severity among South African women during pregnancy and postpartum: population-based prospective cohort study. PLoS Med. 2016;13(1):e1001943.

11. Closson K, et al. Prevalence, type, and correlates of trauma exposure among adolescent men and women in Soweto, South Africa: implications for HIV prevention. BMC Public Health. 2016;16(1):1191.

12. Haffejee $F$, et al. Factors associated with unintended pregnancy among women attending a public health facility in KwaZulu-Natal, South Africa. S Afr Fam Pract. 2018;60(3):1-5.

13. Prioreschi A, et al. Understanding how maternal social and biological factors are related to fetal growth in an urban South African cohort. J Dev Orig Health Dis. 2020;12(2):1-9.

14. Rasty R, Pouraram H, Heshmat R. Food insecurity and some demographic and socioeconomic characteristics, fertility, and pregnancy in women with planned and unplanned pregnancy. Iran J Epidemiol. 2015;11(3):34-42. 
15. Abrahams Z, et al. Factors associated with household food insecurity and depression in pregnant South African women from a low socio-economic setting: a cross-sectional study. Soc Psychiatry Psychiatr Epidemiol. 2018;53(4):363-72.

16. Draper C, et al. Urban young women's preferences for intervention strategies to promote physical and mental health preconception: a healthy life trajectories initiative (HeLTI). Prev Med Rep. 2019;14:100846.

17. Grobler L, Visser M, Siegfried N. Healthy life trajectories initiative: summary of the evidence base for pregnancy-related interventions to prevent overweight and obesity in children. Obes Rev. 2019;20:18-30.

18. Ware LJ, et al. Environmental, social, and structural constraints for health behavior: perceptions of young urban black women during the preconception period-a healthy life trajectories initiative. J Nutr Educ Behav. 2019;51(8):946-57.

19. Hartigan J, Wong M. Algorithm AS 136: a K-means clustering algorithm. J Roy Stat Soc. 1979;28:100-8.

20. Kalsbeek WD, Bowling JM, Hsia J, Mirza S, Palipudi KM, Asma S. The global adult tobacco survey (GATS): sample design and related methods. In Proceedings of the section on survey methods, joint statistical meetings. Alexandria: American Statistical Association 2010 Aug (pp. 3082-96).

21. Saunders JB, et al. Development of the alcohol use disorders identification test (AUDIT): WHO collaborative project on early detection of persons with harmful alcohol consumption-II. Addiction. 1993;88(6):791-804.

22. Murphy A, et al. Adverse childhood experiences (ACEs) questionnaire and adult attachment interview (AAI): implications for parent child relationships. Child Abuse Negl. 2014;38(2):224-33.

23. Kroenke $\mathrm{K}$, et al. The patient health questionnaire somatic, anxiety, and depressive symptom scales: a systematic review. Gen Hosp Psychiatry. 2010;32(4):345-59.

24. Wehler CA, Scott RI, Anderson JJ. The community childhood hunger identification project: a model of domestic hunger-demonstration project in Seattle, Washington. J Nutr Educ. 1992;24(1):29S-35S.

25. de Onis $\mathbf{M}$, et al. Measurement and standardization protocols for anthropometry used in the construction of a new international growth reference. Food Nutr Bull. 2004;25(1):S27-36.

26. World Health Organization. Waist circumference and waist-hip ratio: report of a WHO expert consultation, Geneva, 8-11 December 2008. https://apps.who.int/iris/bitstream/handle/10665/44583/ 9789241501491_eng.pdf.

27. Williams B, et al. 2018 ESC/ESH guidelines for the management of arterial hypertension. Eur Heart J. 2018;39(33):3021-104.

28. Harris PA, et al. The REDCap consortium: building an international community of software platform partners. J Biomed Inform. 2019;95:103208.

29. Flanagan BE, et al. A social vulnerability index for disaster management. JHSEM. 2011;8(1):1.

30. Barbarin OA, Khomo N. Indicators of economic status and social capital in South African townships: what do they reveal about the material and social conditions in families of poor children? Childhood. 1997;4(2):193-222.

31. Kabudula CW, et al. Assessing changes in household socioeconomic status in rural South Africa, 2001-2013: a distributional analysis using household asset indicators. Soc Indic Res. 2017;133(3):1047-73.

32. Statistics by Place/City of Johannesburg/Soweto. (2011). Available from http://www.statssa.gov.za/? page_id=4286\&id=11317. Accessed 10 Mar 2020.

33. Comfort L, et al. Reframing disaster policy: the global evolution of vulnerable communities. Glob Environ Chang Part B Environ Hazard. 1999;1(1):39-44.

34. Battersby J. Urban food insecurity in Cape Town, South Africa: an alternative approach to food access. Dev South Afr. 2011;28(4):545-61.

35. Boatemma S, Drimie S, Pereira L. Addressing food and nutrition security in South Africa: a review of policy responses since 2002. African J Agric Resour Econ. 2018;13(311_2019_687):264-79.

36. Abdu J, Kahssay M, Gebremedhin M. Household food insecurity, underweight status, and associated characteristics among women of reproductive age group in Assayita district, Afar Regional State, Ethiopia. J Environ Public Health. 2018;2018:1-8.

37. Isanaka $\mathrm{S}$, et al. Food insecurity is highly prevalent and predicts underweight but not overweight in adults and school children from Bogota, Colombia. J Nutr. 2007;137(12):2747-55. 
38. UNISDR (United Nations International Strategy for Disaster Reduction). 2015. Sendai framework for disaster risk reduction 2015-30. Available from http://www.wcdrr.org/uploads/Sendai_Frame work_for_Disaster_Risk_Reduction_2015-2030.pdf. Accessed 15 Sep 2019.

39. Kim AW, et al. Idioms of resilience among cancer patients in urban South Africa: an anthropological heuristic for the study of culture and resilience. Transcult Psychiatry. 2019;56(4):720-47.

40. Kohrt BA, et al. Cultural concepts of distress and psychiatric disorders: literature review and research recommendations for global mental health epidemiology. Int J Epidemiol. 2014;43(2):365-406.

41. Madeod C. The causes of teenage pregnancy: a review of South Africa research. Part 1. South Afr J Psychol. 1999;29(1):1-7.

42. Ziraba AK, Fotso JC, Ochako R. Overweight and obesity in urban Africa: a problem of the rich or the poor? BMC Public Health. 2009;9(1):465.

43. Schulz LC. The Dutch hunger winter and the developmental origins of health and disease. Proc Natl Acad Sci. 2010;107(39):16757-8.

44. Weaver LJ, Hadley C. Moving beyond hunger and nutrition: a systematic review of the evidence linking food insecurity and mental health in developing countries. Ecol Food Nutr. 2009;48(4):263-84.

45. Young SL, et al. Household food insecurity, maternal nutritional status, and infant feeding practices among HIV-infected Ugandan women receiving combination antiretroviral therapy. Matern Child Health J. 2014;18(9):2044-53.

46. Lenz M, Richter T, Mühlhauser I. The morbidity and mortality associated with overweight and obesity in adulthood: a systematic review. Dtsch Arztebl Int. 2009;106(40):641.

47. Mabala R. From HIV prevention to HIV protection: addressing the vulnerability of girls and young women in urban areas. Environ Urban. 2006;18(2):407-32.

48. Blaikie P, et al. At risk: natural hazards, people's vulnerability and disasters. London: Routledge, Taylor and Francis Group; 1994.

49. Cutter SL. The vulnerability of science and the science of vulnerability. Ann Assoc Am Geogr. 2003;93(1):1-12.

50. Ranchhod V, Daniels RC. Labour market dynamics in the time of COVID-19 in south africa: Evidence from the NIDS-CRAM survey. Policy Paper. SALDRU, University of Cape Town. 2020 Jul.

51. Casale D, Posel D. Gender and the early effects of the Covid-19 crisis in the paid and unpaid economies in south africa. Natl Income Dyn (NIDS)-Coronavirus Rapid Mobile Survey (CRAM) Wave. 2020 Jul 7;1.

52. Supplementary Budget Review 2020. Department: National Treasury. Republic of South Africa. http://www.treasury.gov.za/documents/national\%20budget/2020S/review/FullSBR.pdf. Accessed 24 June 2020.

53. Institute for Economic Justice. COVID-19: South Africa's COVID-19 Rescue Package Scorecard Update - 11 February 2021. https://www.iej.org.za/wp-content/uploads/2021/02/IEJ-COVID-19-\% E2\%80\%93-rescue-scorecard-2021-1.pdf.

54. Spaull N, et al. National income dynamics study (NIDS) - Coronavirus rapid mobile survey (CRAM) wave 3 synthesis report https://cramsurvey.org/wp-content/uploads/2021/02/1.-Spaull-N.Daniels-R.-C-et-al.-2021-NIDS-CRAM-Wave-3-Synthesis-Report.pdf. Accessed 17 Feb 2021.

55. Bhorat H, Oosthuizen M, Stanwix B. Social Assistance Amidst the COVID-19 Epidemic in South Africa: A Policy Assessment. South African Journal of Economics. 2021;89(1):63-81.

56. COVID-19 RESPONSE POLICY BRIEF \#2: towards a safer, more equitable opening of the economy - 23 July 2020. Institute for Economic Justice, South Africa.

57. Davids E, et al. Adolescent girls and young women: policy-to-implementation gaps for addressing sexual and reproductive health needs in South Africa. S Afr Med J. 2020;110(9):855-7.

58. Mathee A. Environment and health in South Africa: gains, losses, and opportunities. J Public Health Policy. 2011;32(1):S37-43.

59. Abdullah F, Naledi T, Nettleship E, Davids EL, Van Leeuw L, Shangase S, Ramburuth M, Majola N, Dudley L, Nyirenda M, Mathews C. First social impact bond for the SAMRC: A novel financing strategy to address the health and social challenges facing adolescent girls and young women in South Africa. S Afr Med J. 2019;109(11b):57-62.

60. Draper C, et al. Pilot implementation of Bukhali: a preconception health trial in South Africa. SAGE Open Med. 2020;8:2050312120940542. 
Publisher's Note Springer Nature remains neutral with regard to jurisdictional claims in published maps and institutional affiliations.

Lisa J. Ware MSc, $\mathrm{PhD}$, is an Associate Director at SAMRC-Wits Developmental Pathways for Health Research Unit, Faculty of Health Sciences, University of the Witwatersrand, Johannesburg, Gauteng, South Africa and Senior Researcher at DSI-NRF Centre of Excellence in Human Development, University of the Witwatersrand, Johannesburg, Gauteng, South Africa.

Andrew W. Kim MA, is a PhD Candidate at the Department of Anthropology, Northwestern University, Evanston, Illinois, USA and Honorary Researcher at SAMRC-Wits Developmental Pathways for Health Research Unit, Faculty of Health Sciences, University of the Witwatersrand, Johannesburg, Gauteng, South Africa.

Alessandra Prioreschi PhD, is a Researcher at SAMRC-Wits Developmental Pathways for Health Research Unit, Faculty of Health Sciences, University of the Witwatersrand, Johannesburg, Gauteng, South Africa.

Lukhanyo H. Nyati MSc, ia a Data Scientist at SAMRC-Wits Developmental Pathways for Health Research Unit, Faculty of Health Sciences, University of the Witwatersrand, Johannesburg, Gauteng, South Africa.

Wihan Taljaard BSc Hons IT, is a Data Administrator at SAMRC-Wits Developmental Pathways for Health Research Unit, Faculty of Health Sciences, University of the Witwatersrand, Johannesburg, Gauteng, South Africa.

Catherine E. Draper MA, PhD is Associate Professor at SAMRC-Wits Developmental Pathways for Health Research Unit, Faculty of Health Sciences, University of the Witwatersrand, Johannesburg, Gauteng, South Africa.

Stephen J. Lye $\mathrm{PhD}$ is an Interim Director and Senior Scientist at the Lunenfeld-Tanenbaum Research Institute, Sinai Health and Professor of Obstetrics and Gynaecology, Physiology and Medicine, University of Toronto, ON, Canada.

Shane A. Norris PhD is a Professor and Director at SAMRC-Wits Developmental Pathways for Health Research Unit, Faculty of Health Sciences, University of the Witwatersrand, Johannesburg, Gauteng, South Africa and Professorial Fellow at the Global Health Research Institute, School of Human Development and Health \& NIHR Southampton Biomedical Research Centre, University of Southampton, UK.

\section{Authors and Affiliations}

\section{Lisa J. Ware ${ }^{1,2}$ (1) . Andrew W. Kim ${ }^{1,3} \cdot$ Alessandra Prioreschi $^{1}$. Lukhanyo H. Nyati ${ }^{1}$. Wihan Taljaard ${ }^{1}$. Catherine E. Draper ${ }^{1}$. Stephen J. Lye ${ }^{4,5}$. Shane A. Norris ${ }^{1,6}$}

1 SAMRC-Wits Developmental Pathways for Health Research Unit, Faculty of Health Sciences, Corner College and Clinic Roads, Chris Hani Baragwanath Academic Hospital, University of the Witwatersrand, Soweto, Johannesburg, Gauteng, South Africa

2 DSI-NRF Centre of Excellence in Human Development, University of the Witwatersrand, Johannesburg, Gauteng, South Africa

3 Department of Anthropology, Northwestern University, Evanston, IL, USA

4 Departments of Physiology, Obstetrics and Gynaecology, and Medicine, University of Toronto, 


\section{Toronto, ON, Canada}

5 Lunenfeld-Tanenbaum Research Institute, Sinai Health, Toronto, ON, Canada

6 School of Human Development and Health and NIHR Southampton Biomedical Research Centre, Global Health Research Institute, University of Southampton, Southampton, UK 